\title{
Ecosystem approaches to controlling of vector-borne diseases: dengue and Chagas disease
}

Worldwide concern by international, national and local institutions about environmental degradation, quality of life, and poverty have emerged in recent decades. This focus has been expressed in public policies and interventions aimed at mitigating the precarious living conditions in deteriorated ecosystems, especially in developing countries. Yet the complexity and interdependence among factors related to health, environment, and economics require participatory methodologies to produce comprehensive, integrated knowledge that allows the implementation of effective inter-sector actions.

In this context, the Ecohealth program initiative of Canada's International Development Research Centre (IDRC; http://www.idrc.ca/ecohealth) has supported scientific research with systemic approaches based on an integrated multidisciplinary academic perspective combined with local and participatory experiences. The aim is to produce transdisciplinary knowledge for utilization on ecosystem management to improve quality of life in sustainable ecosystems. In order to disseminate these research results, the current Supplement of Cadernos de Saúde Pública/Reports in Public Health presents articles that originated from projects supported by the IDRC/Ecohealth, focusing on two highly relevant diseases for public health, Chagas disease and dengue, both characterized by vector transmission. The articles on projects with ecosystem approaches include participation of different stakeholders, from health, education, engineering, and sanitation sectors, with various levels of involvement in knowledge output and implementation of pertinent actions.

The review articles demonstrate the influence of ecological changes on the determination of Chagas disease, which was initially limited almost entirely to rural areas, but which has now become a potential problem in urban ecosystems affected by various processes such as deforestation, migration, and marketing of certain products contaminated with the etiological agent. The review articles also call attention to dengue, an arbovirus infection that has reemerged with surprising force since the 1980s often in large metropolises, especially in the Americas, producing explosive, large scale epidemics. The complexity and diversity of factors involved in the solution to these problems highlight the need to integrate knowledge and involve different stakeholders that can lead local projects to improve human health conditions. Such initiatives represent a relevant contribution to the implementation of the global agenda for sustainable development.

Maria Glória Teixeira

Instituto de Saúde Coletiva, Universidade Federal da Bahia,

Salvador, Brasil.

magloria@ufba.br

Ana Boischio

The International Development Research Centre, Ottawa, Canada.

aboischio@idrc.ca

Maria da Conceição N. Costa

Instituto de Saúde Coletiva, Universidade Federal da Bahia,

Salvador, Brasil.

mcncosta@ufba.br 